\title{
Targeting the Metabolism and Receptors of Sphingosine-1-Phosphate for the Treatment of Rheumatoid Arthritis
}

\author{
Sylvain G. Bourgoin, Chenqi Zhao \\ and Maria J. Fernandes \\ Laval University \\ Canada
}

\section{Introduction}

Sphingolipids belong to a major class of lipids that are ubiquitously synthesized by eukaryotic cells and are characterized by their sphingoid backbone and primary structural roles in membrane formation. Over the past two decades, sphingolipids have emerged as a source of important signalling molecules in addition to their structural functions (Merrill et al., 1997). In particular, sphingosine-1-phosphate (S1P), is a unique signalling molecule that has the ability to act as an intracellular second messenger, as well as an extracellular stimulus through specific G protein-coupled receptor (GPCRs) (Pyne and Pyne, 2000; Spiegel and Milstien, 2003; Van Brocklyn et al., 1998). S1P has been shown to mediate a variety of fundamental biological processes including cell proliferation, migration, invasion, angiogenesis, vascular maturation and lymphocyte trafficking. The intracellular level of S1P is tightly regulated by the equilibrium between its synthesis by sphingosine kinases (SphKs) and its degradation by S1P phosphatases (SPPs) and S1P lyase (SPL) (Saba and Hla, 2004). Activation of SphKs by a variety of agonists, including growth factors, cytokines and hormones, increases intracellular S1P. Once generated, S1P can either act intracellularly as a second messenger or be secreted out of the cell and act extracellularly by binding to and signalling through S1P receptors expressed on the surface of the same cell or nearby cells in autocrine and/or paracrine manners (Alvarez et al., 2007). Many of the biological effects of S1P are mediated by its binding to and activation of its specific receptors S1P1-S1P5 (Ishii et al., 2004). Alterations in S1P signalling, as well as in the enzymes involved in its synthesis and metabolism, have been observed in many types of pathological situations such as angiogenesis, metastasis, and autoimmunity.

The role played by S1P in the autoimmune disease rheumatoid arthritis (RA) has recently emerged. Activated SphK and elevated levels of S1P have been detected in the synovium and synovial fluids of patients with RA, respectively (Kamada et al., 2009; Kitano et al., 2006; Lai et al., 2008). In addition, exogenously applied S1P in cultured synovial fibroblasts from RA patients causes cell migration, production of cytokines/chemokines, expression of cyclooxygenase- 2 and prostaglandins, as well as cell proliferation and survival (Kitano et al., 2006; Zhao et al., 2008). These results suggest a biological function for the SphK/S1P/S1P receptor (S1PR) axes in RA disease progression. In this review we summarize the current understanding of how S1P metabolism and signalling impact the biology of inflammation in 
RA. We also discuss the potential therapeutic benefit of modulators of S1P metabolism and S1P receptors, including SphK/SPL inhibitors, FTY-720, S1P receptor antagonists, and antiS1P monoclonal antibodies, in the treatment of RA.

\section{S1P biosynthesis, metabolism and secretion}

All cells are able to generate S1P during the normal physiologic metabolism of sphingolipids (Hla, 2004). Generally, systemic and local concentrations of S1P are tightly regulated by the balance between its synthesis and degradation via three enzyme families, including SphKs (SphK1 and SphK2), SPPs (SPP1 and SPP2), and SPL (Fig. 1). SphKs generate S1P through phosphorylation of its precursor, sphingosine, while SPPs reversibly convert S1P back to sphingosine by removing the phosphate (Xia et al., 2000). Irreversible degradation of S1P is carried out by a single enzyme SPL, which cleaves S1P into ethanolamine phosphate and hexadecenal, representing the last step in the sphingolipid degradation pathway (Xia et al., 2000).

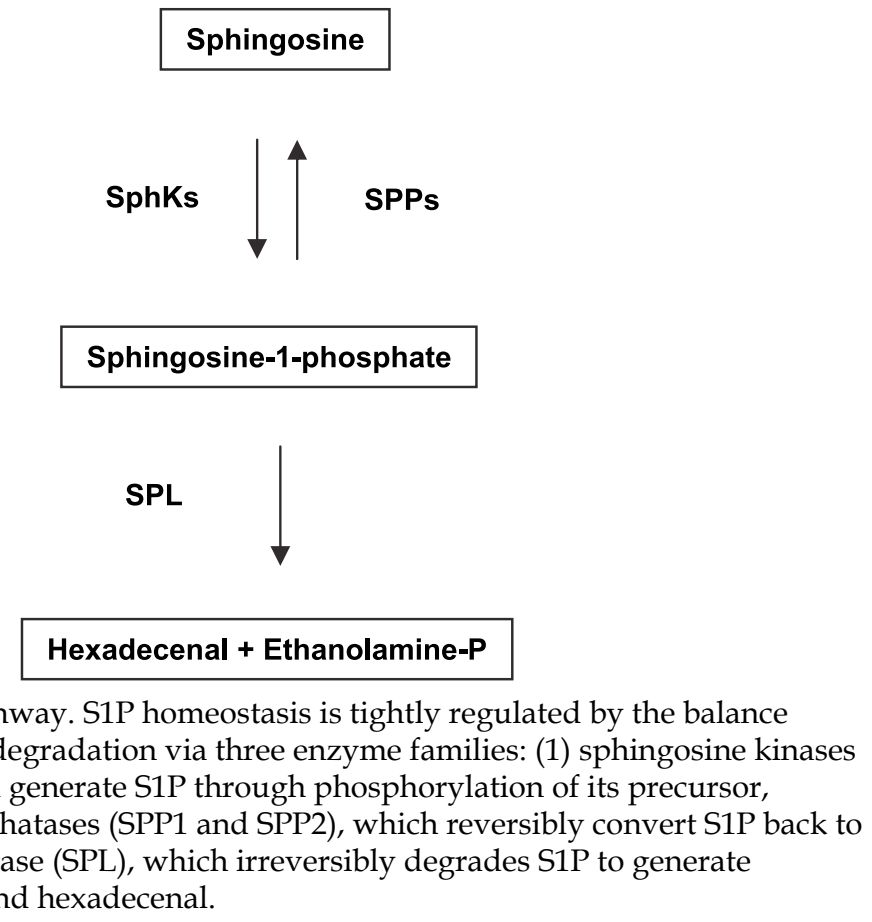

SphK is represented by two different isoforms (SphK1 and SphK2). Although both SphK1 and SphK2 can phosphorylate sphingosine, SphK1 produces the majority of S1P in cells (Kohama et al., 1998). SphKs can be activated by a large variety of agonists, including growth factors (such as platelet-derived growth factor, vascular endothelial growth factor, epidermal growth factor and hepatocyte growth factor), cytokines (such as TNF- $\alpha$ ), steroid hormones (such as estradiol), and GPCR ligands (such as lysophosphatidic acid (LPA) and S1P) (reviewed in (Alvarez et al., 2007; Spiegel and Milstien, 2003; Taha et al., 2006)). Following cellular activation by these agonists, SphK1 can be activated through: 1) 
phosphorylation and translocation to the plasma membrane, 2) interaction with acidic phospholipids such as phosphatidic acid produced by phospholipase D (Delon et al., 2004), and 3) possible association with other proteins. All or parts of these mechanisms may be required for full activation of SphK1 (Takabe et al., 2008). Phosphorylation of SphK1 leads to its translocation to the plasma membrane where its substrate sphingosine is located, resulting in the production of S1P (Johnson et al., 2002; Pitson et al., 2005). S1P, in turn, activates specific S1P receptors present on the surface of the same cell or on nearby cells in autocrine and/or paracrine manners (Alvarez et al., 2007). The "inside-out" export of intracellularly generated S1P is crucial for many S1P functions (Takabe et al., 2008).

The mechanism by which S1P is exported from the inside to the outside of cells after synthesis is not fully understood. Several lines of evidence suggest the involvement of the ATP-binding cassette (ABC) family of transporters in S1P secretion (Kobayashi et al., 2006; Mitra et al., 2006; Sato et al., 2007). Mitra et al. (2006) revealed that the release of S1P from mast cells is regulated by ABCC1. Similarly, the ABCA1 transporter is critical for the release of S1P from astrocytes (Sato et al., 2007). In breast cancer the export of S1P mediated by $\mathrm{ABCC} 1$ and ABCG2 transporters is stimulated by estrogen receptor- $\alpha$ (Takabe et al., 2010). Altogether, these studies suggest that members of the family of $A B C$ transporters may be important for export of S1P out of cells.

\section{S1P receptors and downstream signalling pathways}

S1P evokes diverse biological functions by binding to its ubiquitously-expressed and specific cell surface receptors. So far, five S1P receptors, designated as S1P1/Edg1, S1P2/Edg5, S1P3/Edg3, S1P4/Edg6, and S1P5/Edg8, have been identified (An et al., 1997; Im et al., 2000; Lee et al., 1998; Van Brocklyn et al., 2000; Yamazaki et al., 2000). These receptors were initially named "Edg" receptors as they belong to the so-called endothelial differentiation gene (Edg) family; however "S1P receptors" is now preferred (Chun et al., 2002). S1P receptors are GPCRs that share high similarity with each other and with LPA receptors. S1P receptors exhibit variable tissue distribution, for example, S1P1, S1P2, and S1P3 are widely expressed in various tissues, whereas the expression of S1P4 and S1P5 is more restricted to cells of the immune system and nervous system, respectively (reviewed in (Sanchez and Hla, 2004)). Upon binding to S1P receptors, S1P activates downstream signalling pathways, leading to a variety of cellular responses such as proliferation, cell migration, actin cytoskeletal rearrangement, and adherens junction assembly (Kluk and Hla, 2002; Taha et al., 2004). Each S1P receptor is coupled to a specific heterotrimeric G protein $\left(G_{i / o}, G_{q}\right.$ and $\left.G_{12 / 13}\right)$, which, when activated, dissociates into its $\alpha$ and $\beta \gamma$ subunits and transduces signals toward the downstream pathways. Particularly, S1P1 is coupled predominantly to $G_{i / o}$, through which it activates: 1) Rho kinase and tyrosine kinases, leading to cytoskeletal rearrangement (Garcia et al., 2001); 2) MAPK, leading to angiogenesis (Lee et al., 1999); and 3) Akt, leading to cell chemotaxis (Lee et al., 2001). On the other hand, S1P2 and S1P3 are linked predominantly to $\mathrm{G}_{\mathrm{q}}$ and $\mathrm{G}_{12 / 13}$, through which they activate phospholipase $\mathrm{C}$ leading to $\mathrm{Ca}^{2+}$ mobilization (Ancellin and Hla, 1999) and positive or negative modulation of Rho and thus of cell migration (Sugimoto et al., 2003). More detailed information on the various signalling pathways turned on by S1P receptor activation is discussed in previous reviews (Choi et al., 2008; Rosen et al., 2009).

It is known that S1P can also act intracellularly to enhance cell proliferation and suppress apoptosis independently of S1P receptors (Rosenfeldt et al., 2001; Van Brocklyn et al., 1998). In plants and yeast, which do not express S1P receptors, for instance, intracellular S1P 
regulates stomatal aperture (Coursol et al., 2005) or stress responses and survival (Jenkins and Hannun, 2001), respectively. Similarly, in mammalian cells intracellular S1P regulates calcium release independently of inositol trisphosphate formation and of S1P receptor activation (Blom et al., 2005). S1P also signals within the nucleus by binding to and inhibiting histone deacetylases HDAC1 and HDAC2, leading to the epigenetic regulation of gene expression (Hait et al., 2009). Further studies are needed, however, to reveal the intracellular second messenger functions of S1P.

\section{Role of SphK/S1P/S1PR signalling in rheumatoid arthritis}

\subsection{Rheumatoid arthritis}

Rheumatoid arthritis (RA) is an autoimmune disease characterized by chronic inflammation of the joint synovium, synovial hyperplasia and a massive infiltration of inflammatory cells into the affected joints, leading to the progressive destruction of cartilage and bone (Feldmann et al., 1996a). The inciting agent that triggers the development of RA is unknown; however, the disease is clearly an inflammatory process since the critical events in RA are largely coordinated by complex interplays of proinflammatory cytokines, chemokines, and matrix metalloproteinases produced by both synovial resident cells and infiltrating cells (Feldmann et al., 1996b; McInnes and Schett, 2007). These inflammatory mediators are involved in many pathological processes in RA. Increasing evidence demonstrates,

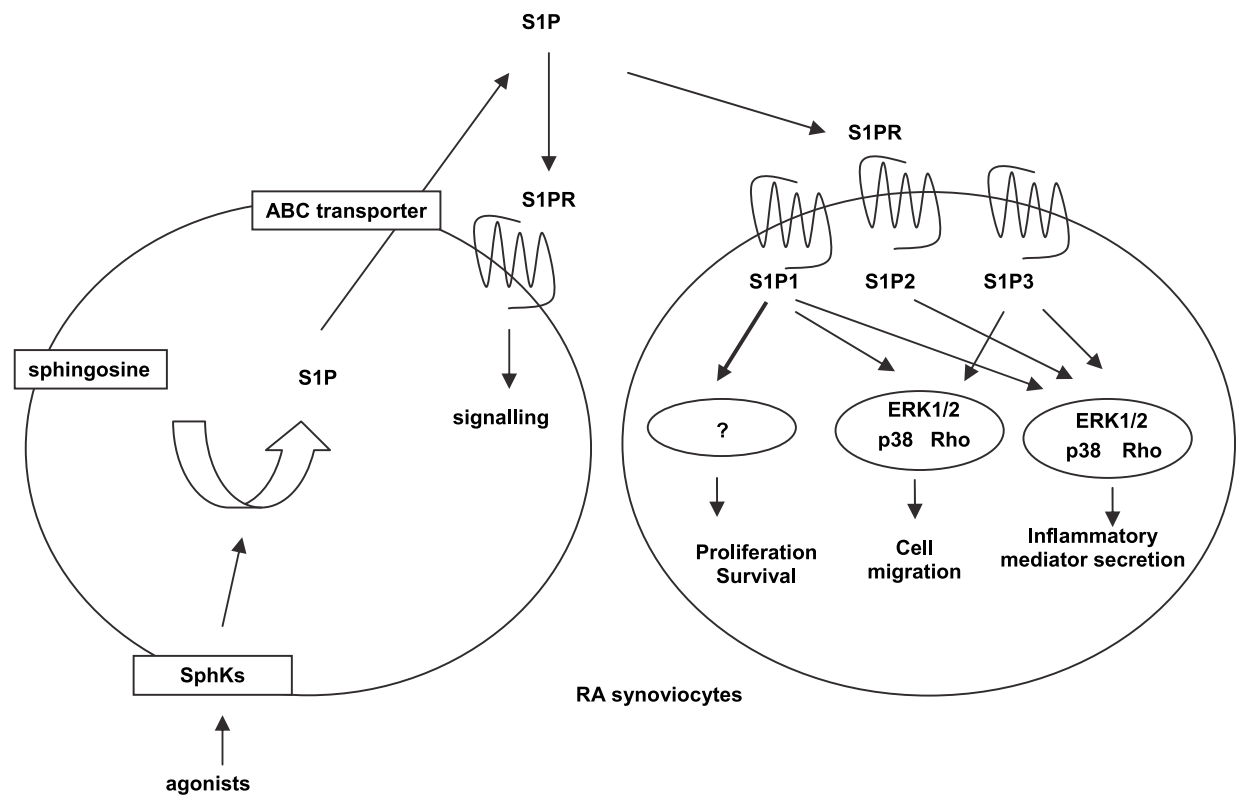

Fig. 2. S1P metabolism and signalling in RA synoviocytes. A range of agonists including growth factors, hormones, angiogenic factors and other stimuli activate SphKs through phosphorylation. S1P is then generated and exported out of the cells by ABC transporters to act on the same cell (autocrine) or on nearby cells (paracrine). S1P engages with its receptors (S1P1-3) to mediate a diverse array of signalling pathways, impacting fundamental biological processes that are integral to the pathogenesis of RA, such as cell proliferation and survival, cell migration, and inflammatory mediator secretion. 
however, that the activation of RA synoviocytes can be maintained in the absence of inflammatory cytokines (Muller-Ladner et al., 1996), indicating the involvement of biologically active mediators other than inflammatory cytokines in RA disease progression. Emerging evidence suggests a role for S1P metabolism and signalling in various aspects of the pathogenesis of RA. Recent in vitro and in vivo studies evaluated the potential role of S1P synthesis, catabolism enzymes and S1P signalling in RA (Fig. 2).

\subsection{S1P expression and SphK activity in RA synovium and synovial fluids}

Several studies reveal high levels of S1P in RA synovial tissues and fluids (Kitano et al., 2006; Lai et al., 2008). Kitano et al. (2006) reported elevated levels of S1P in synovial fluids of RA patients $(1078 \mathrm{pM} / \mathrm{ml})$ in comparison to those of osteoarthritis (OA) patients (765 $\mathrm{pM} / \mathrm{ml}$ ). The S1P content in RA synovial fluids was significantly higher than those in serum $(400 \mathrm{pM} / \mathrm{ml})$ or plasma $(100 \mathrm{pM} / \mathrm{ml})$ from normal people. Lai et al. (2008) also measured the amount of S1P by a competitive ELISA and detected up to $17 \mu \mathrm{M}$ S1P in RA synovial fluids, which was more than five fold higher than that found in OA synovial fluids $(3 \mu \mathrm{M})$. The increased level of S1P could be responsible for the recruitment and the infiltration of immune cells into the synovium (see section 4.2.2). Pi et al. (2006) also showed that peripheral blood B lymphoblastoid cell lines derived from patients with RA exhibit a high level of S1P synthesis.

SphK expression and activity were also demonstrated in RA. Increased SphK1 expression and activity were found in RA B lymphoblastoid cell lines, which were implicated as the underlying mechanism of impaired Fas-mediated death signalling in RA (Pi et al., 2006). More recently, SphK2 was shown to be expressed in rheumatoid synovial fibroblasts in vivo and in vitro and associated with the up-regulation of S1P production (Kamada et al., 2009). Surprisingly, FTY-720 (2-amino-2-[2-(4-octylphenyl) ethyl] propane-1, 3-diol hydrochloride), a sphingosine analog that is phosphorylated to the active metabolite FTY-720-phosphate (FTY-720-P) by SphK2 (Brinkmann et al., 2002; Mandala et al., 2002), induces apoptosis of synovial fibroblasts. This effect could be due, at least in part, to FTY-720-P-mediated degradation of specific S1P receptors (Matloubian et al., 2004). Corroborating this observation, we reported that S1P protects synovial fibroblasts from ongoing apoptosis (Zhao et al., 2008). Using the mouse model of collagen-induced arthritis (CIA), Lai et al. (2009) highlighted distinct roles for SphK1 and SphK2 in regulating cell growth and survival. The in vivo administration of SphK1 siRNA reduced inflammation whereas mice treated with SphK2 siRNA resulted in a more aggressive disease and greater secretion of proinflammatory cytokines (IL-6, TNF- $\alpha$ and IFN- $\alpha$ ) by immune cells. How and why SphK1 and SphK2 exert distinct opposing roles in the regulation of inflammatory arthritis remains unclear and may be related to the location of S1P production (Maceyka et al., 2005). The role of SphK2 in chronic inflammatory diseases remains ambiguous since the selective inhibitor of SphK2 ABC249640 has been reported to reduce bone and cartilage degradation in the mouse models of adjuvant-induced arthritis (AIA) and of CIA (Fitzpatrick et al., 2011). The SphK1 signalling pathway is activated in RA (Limaye et al., 2009). In the mouse model of CIA, administration of a non-specific pharmacological inhibitor of SphKs, N,Ndimethylsphingosine (DMS), and a siRNA approach to knockdown the SphK1 isoform, markedly suppressed joint pathologies such as adjacent cartilage and bone erosion, synovial hyperplasia, and infiltration of inflammatory cells into the joint compartment (Lai et al., 2008). Moreover, SphK1 deficiency in hTNF- $\alpha$ transgenic mice that develop arthritis at an early age was related to less synovial inflammation and bone erosion (Baker et al., 2010). To 
our knowledge the effect of specific inhibitors of SphK1, such as BML-258 (Paugh et al., 2008), has not been tested in animal models of arthritis.

At present, evidence for roles of SPPs and SPL in RA is limited. However, up-regulation of SPP2 was detected in samples of skin lesions from patients with psoriasis, a chronic inflammatory skin disease (Mechtcheriakova et al., 2007). Interestingly, elevated mRNA expression of SPP1 and SPL was observed in RA synoviocytes compared to non-arthritic synoviocytes (Zhao et al., unpublished data).

\subsection{S1P receptor expression in RA synovial fibroblasts}

In RA, the abnormal growth and erosive activity of synovial fibroblasts suggest that these cells are important contributors to chronic inflammation. During RA disease progression, synovial fibroblasts become hyperplasic and closely interact with infiltrated immune cells to form the aggressive pannus tissue that eventually promotes joint destruction (reviewed in (Feldmann et al., 1996a)). Synovial fibroblasts have been reported to express three of five known S1P receptors, S1P1-3 (Kitano et al., 2006; Nochi et al., 2008; Zhao et al., 2008). Expression of S1P1 in RA synovial tissues was significantly higher compared to that of OA synovial tissues (Kitano et al., 2006). Moreover, pretreatment of synovial fibroblasts with TNF- $\alpha$, the well-recognized critical cytokine in RA, resulted in up-regulation of S1P3 receptor expression in synovial fibroblasts, which likely contributes to the synergistic production of inflammatory cytokines/chemokines upon subsequent exposure to S1P (Zhao et al., 2008).

\subsection{S1P signalling in RA pathophysiology}

\subsubsection{S1P signalling in migration of synovial fibroblasts and osteoclasts}

As constituents of the synovial pannus in RA, synovial fibroblasts have long been considered as key players in the aggressive destruction of cartilage and bone (Shiozawa et al., 1983). Using an in vitro wound-closing assay, we previously reported that S1P could induce synoviocyte migration (Zhao et al., 2008). S1P-induced cell migration is mediated through the activation of extracellular signal-regulated kinase-1 and -2 (ERK1/2), as well as p38 mitogen-activated protein kinase (MAPK) and of Rho kinase. S1P-driven motility of synoviocytes was mainly via S1P1 and S1P3 receptor activation since the effect of S1P was mimicked by the S1P1 receptor agonist (SEW2871) but abolished by S1P1/3 (VPC23019) and S1P3 (CAY10444) receptor antagonists.

$\mathrm{S} 1 \mathrm{P}$ has also been shown to induce chemotaxis and regulate migration of osteoclasts and their precursors in vitro and in vivo (Ishii et al., 2009). Cells bearing the properties of osteoclast precursors express S1P1 receptors and migrate along an S1P gradient in vitro. The S1P1 agonist, SEW2871, stimulated motility of osteoclast precursors-containing monocytoid populations in vivo. In addition, osteoclast/monocyte lineage-specific conditional S1P1 knockout mice show osteoporotic changes due to increased osteoclast attachment to the bone surface, suggesting a crucial role of the S1P-S1P1 receptor axis in regulating the migratory behaviour of osteoclast precursors and bone mineral homeostasis.

\subsubsection{S1P signalling in the secretion of cytokines/chemokines and lipid mediators}

In the inflamed RA synovium, large amounts of pro-inflammatory cytokines and chemokines may contribute directly to cartilage and bone erosion by promoting matrix metalloproteinase (MMP) production and suppressing chondrocyte/osteoclast functions (Feldmann et al., 1996b). Previous studies from our group and others demonstrated that exogenously applied S1P could stimulate synovial fibroblasts to release various 
inflammatory mediators, including cytokines, chemokines, and prostaglandin $\mathrm{E}_{2}\left(\mathrm{PGE}_{2}\right)$ (Kitano et al., 2006; Zhao et al., 2008). S1P administration strongly stimulated the secretion of IL-8 (interleukin-8), IL-6, MCP-1 (monocyte chemotactic protein-1), and RANTES (regulated on activation normal $\mathrm{T}$ cells expressed and secreted) via S1P2 and S1P3 receptors. The signalling pathways involved in S1P-mediated cytokine/chemokine secretion were dependent on ERK1/2, p38 MAPK, and Rho kinase activation. These cytokines/chemokines may subsequently increase the recruitment of inflammatory cells such as neutrophils into the synovium, as chemokines such as IL-8 exhibit selective chemotactic activity for neutrophils, whereas MCP-1, MIP-1 $\alpha$ (macrophage inflammatory protein-1 $\alpha$ ), MIP-1 $\beta$ (macrophage inflammatory protein-1 $\beta$ ), and RANTES primarily attract monocytes (Koch, 2005). S1P may therefore contribute to the regulation and maintenance of the inflammatory response in RA, in part through stimulation of multiple cytokine/chemokine secretion by synovial fibroblasts. SphK/S1P signalling was also implicated in cell-contact-mediated proinflammatory cytokine/chemokine secretion in RA synovium without additional exogenous stimulation. Lai et al. (2008) used peripheral blood mononuclear cells from RA patients in cell-cell contact experiments. They discovered that activated peripheral $\mathrm{T}$ lymphocytes from RA patients induced substantial production of TNF- $\alpha$, IL-1 $\beta$, IL-6, MCP-1 and MMP-9 by autologous peripheral monocytes. Importantly, treatment with a potent SphK inhibitor, DMS, significantly suppressed production of these cytokines. The results suggest the importance of SphK/S1P signalling in cell-contact-mediated inflammatory mediators, which is relevant to RA pathology.

Regarding $\mathrm{PGE}_{2}, \mathrm{~S} 1 \mathrm{P}$ was reported to stimulate COX-2 expression and super-production of $\mathrm{PGE}_{2}$ by RA synovial fibroblasts (Kitano et al., 2006; Nochi et al., 2008). $\mathrm{PGE}_{2}$ is an autocrine lipid mediator derived from arachidonic acid metabolism by cyclooxygenases (COX-1 or COX2) (Ghosh et al., 2004). High levels of $\mathrm{PGE}_{2}$ were detected in synovial fluids of RA patients (Hidaka et al., 2001; Lettesjo et al., 1998). PGE $_{2}$ has pro-inflammatory properties and contributes to the pathogenesis of arthritis, since $\mathrm{PGE}_{2}$ stimulates angiogenesis in the rheumatoid synovium (Ben-Av et al., 1995) and triggers bone resorption by osteoclasts (Mino et al., 1998). Thus, S1P may aggravate synovial hyperplasia, inflammation and angiogenesis through the induction of COX-2 and $\mathrm{PGE}_{2}$ in RA synovial tissues. Indeed, the COX-2-PGE 2 axis has been suggested to play an important role in arthritic diseases by stimulating inflammation, angiogenesis, and osteoclastic bone destruction (Martel-Pelletier et al., 2003).

\subsubsection{S1P signalling in the proliferation and survival of RA synovial fibroblasts, B lymphoblastoid cells and chondrocytes}

Abnormal proliferation and resistance to apoptosis of synovial fibroblasts have been suggested to contribute directly to the hyperplasia of the rheumatoid synovium (Ospelt et al., 2004). Indeed, S1P appears capable of increasing cell survival and inhibiting apoptosis of various cell types, including B lymphoblastoid cells derived from RA patients (Pi et al., 2006). We previously demonstrated that S1P, through S1P1, protected synovial fibroblasts from apoptosis (Zhao et al., 2008). In our study we were not able to demonstrate a significant effect of S1P on RA synoviocyte proliferation, although Kitano et al. (2006) reported a small stimulatory effect of S1P on cell proliferation. Nonetheless these studies suggest that S1P can induce the proliferation of synovial fibroblasts, which may contribute to synovial hyperplasia (Knedla et al., 2007). S1P was also reported to aggravate arthritis by inducing chondrocyte proliferation through the stimulation of COX-2 and $\mathrm{PGE}_{2}$ production and via the activation of ERK (Kim et al., 2006; Masuko et al., 2007). 


\subsubsection{Synergistic action of S1P and TNF- $\alpha$ on synovial fibroblast functions}

TNF- $\alpha$ is central to RA pathogenesis as the pro-inflammatory cytokine not only stimulates the production of other inflammatory mediators but also directly triggers the activation of synovial cells and osteoclasts leading to the irreversible damage of soft tissues and bone (Olsen and Stein, 2004). Several studies have suggested that S1P and TNF- $\alpha$ might synergize in their regulation of synovial fibroblast functions. On the one hand, the expression of S1P3 receptor was enhanced by TNF- $\alpha$, leading to amplified secretion of cytokines/chemokines, including IL-6, IL-8, MCP-1, and RANTES (Zhao et al., 2008). On the other hand, S1P enhanced TNF- $\alpha$-mediated COX-2 expression and production of $\mathrm{PGE}_{2}$ by RA synoviocytes (Kitano et al., 2006). Thus, the elevated levels of TNF- $\alpha$ seen in RA synovium may increase S1P synthesis via activation of SphKs and make synovial fibroblasts more responsive to S1P possibly through up-regulation of S1P receptor expression. The synergy between TNF- $\alpha$ and S1P may eventually exacerbate the clinical manifestations of RA, including enhanced synovial tissue invasion by aggressive fibroblasts (due to enhanced cell mobility), synovial hyperplasia (due to proliferation and survival of synoviocytes), and exacerbated inflammation (due to over production of inflammatory mediators).

\section{Potential S1P-targeted therapy for RA}

Despite great efforts devoted to the development of new therapeutic targets for RA, currently used drugs have limitations in their use for the treatment of RA. As discussed in the above sections, S1P appears to be an important modulator of many aspects of the pathogenesis of RA. Manipulation of endogenous local and systemic amounts of bioactive S1P by inhibiting metabolic enzymes such as SphKs and SPL, by applying S1P-blocking agents such as FTY-720, VPC23019 and JTE-013 or anti-S1P antibodies is thus a promising approach for the treatment of RA. Studies targeting S1P metabolism and signalling for the treatment of RA are summarized in Table 1.

\subsection{Targeting S1P metabolism}

\subsubsection{Reducing S1P formation with sphingosine kinase inhibitors}

Blockade of SphK1 activity in the mouse model of CIA significantly suppressed articular inflammation and joint destruction, reduced disease severity, and down-regulated proinflammatory cytokine production and inflammatory cell recruitment into the synovium (Lai et al., 2009). Decreasing S1P production by inhibiting SphK activity is, therefore, a promising therapeutic option in chronic inflammatory arthritis. Indeed, the inhibition of S1P synthesis by blocking SphKs activity has proven useful as an anti-inflammation strategy in cancer therapy in vitro and in animal models (Billich et al., 2005; Bonhoure et al., 2006; French et al., 2006; Gamble et al., 2006; Leroux et al., 2007).

\subsubsection{Downregulation of S1P receptor activity with S1P lyase inhibitors}

SPL catalyzes the irreversible degradation of intracellular S1P to hexadecenal and phosphoethanolamine. It is the major and constitutively active S1P-degrading enzyme in cells and tissues (Fig. 1). As a result, S1P concentrations in tissues are maintained at very low levels. Inhibition of SPL leads to the accumulation of S1P in various tissues (Schwab et al., 2005). Treatment with SPL inhibitor results in accumulation of S1P in lymphoid tissues 
Targeting the Metabolism and Receptors of

\begin{tabular}{|c|c|c|c|c|}
\hline $\begin{array}{l}\text { Approach and } \\
\text { reagent }\end{array}$ & $\begin{array}{l}\text { Mechanism of } \\
\text { action }\end{array}$ & Target & $\begin{array}{l}\text { Experimental } \\
\text { system }\end{array}$ & Reference \\
\hline \multicolumn{5}{|l|}{$\begin{array}{l}\text { Inhibition of } \\
\text { SphK activity }\end{array}$} \\
\hline $\begin{array}{l}N, N- \\
\text { dimethylsphingo } \\
\text { sine (DMS, SphK } \\
\text { inhibitor) }\end{array}$ & $\begin{array}{l}\text { Reduces S1P } \\
\text { formation }\end{array}$ & $\begin{array}{l}\text { SphK1 and } \\
\text { SphK2 }\end{array}$ & In vivo & Lai et al., 2008 \\
\hline $\begin{array}{l}\text { ABC249640 } \\
\text { (SphK2 inhibitor) }\end{array}$ & $\begin{array}{l}\text { Reduces S1P } \\
\text { formation }\end{array}$ & SphK2 & In vivo & $\begin{array}{l}\text { Fitzpatrick et al., } \\
2011\end{array}$ \\
\hline siSphK1 & $\begin{array}{l}\text { Reduces S1P } \\
\text { formation }\end{array}$ & SphK1 & In vivo & Lai et al., 2008 \\
\hline \multicolumn{5}{|c|}{\begin{tabular}{l|l} 
Inhibition of SPL & \\
activity &
\end{tabular}} \\
\hline SPL inhibitor & $\begin{array}{l}\text { Downregulates } \\
\text { S1PR activity }\end{array}$ & SPL & Clinical trial & $\begin{array}{l}\text { Bagdanoff et al., } \\
2010\end{array}$ \\
\hline \multicolumn{5}{|l|}{$\begin{array}{l}\text { Sequestration of } \\
\text { S1P }\end{array}$} \\
\hline $\begin{array}{l}\text { S1P specific } \\
\text { antibody }\end{array}$ & \begin{tabular}{|l|} 
Depletes \\
extracellular S1P
\end{tabular} & S1P & In vivo & $\begin{array}{l}\text { Visentin et al., } \\
2006\end{array}$ \\
\hline \multicolumn{5}{|l|}{ Blocking S1PR } \\
\hline $\begin{array}{l}\text { FTY-720 } \\
\text { (sphingosine } \\
\text { analogue) }\end{array}$ & $\begin{array}{l}\text { Down-regulates } \\
\text { S1PRs and } \\
\text { renders cells } \\
\text { unresponsive to } \\
\text { S1P }\end{array}$ & $\begin{array}{l}\text { S1P1, S1P3, S1P4 } \\
\text { and S1P5 }\end{array}$ & In vivo & $\begin{array}{l}\text { Matsuura et al., } \\
\text { 2000; Tsunemi et } \\
\text { al.; Wang et al., } \\
2007\end{array}$ \\
\hline $\begin{array}{l}\text { VPC23019 } \\
\text { (S1P1/3 } \\
\text { antagonist), } \\
\text { CAY10444 (S1P3 } \\
\text { antagonist) and } \\
\text { JTE-013 (S1P2 } \\
\text { antagonist) }\end{array}$ & $\begin{array}{l}\text { Block S1P } \\
\text { binding to S1PR }\end{array}$ & S1P1-3 & In vitro & $\begin{array}{l}\text { Kitano et al., } \\
2006 \text {; Zhao et al., } \\
2008\end{array}$ \\
\hline
\end{tabular}

Table 1. Targeting the SphK/S1P/S1PR signalling pathway in RA.

and induces premature internalization of the exit-signal-sensing S1P1 receptor on lymphocytes (Schwab et al., 2005). S1P1 receptor internalization renders lymphocytes unresponsive to S1P, preventing their egress from the thymus and lymph nodes (Lo et al., 2005). One physiological outcome of this systemic redistribution of lymphocytes is potent immunosuppression, which offers new opportunities for developing immunoregulatory agents to treat autoimmune and inflammatory diseases (Gardell et al., 2006; Huwiler and Pfeilschifter, 2008; Mandala et al., 2002; Rosen et al., 2003; Zhang and Schluesener, 2007). Indeed, SPL-deficient mice showed resistance to various inflammatory and autoimmune challenges (Bagdanoff et al., 2009; Bandhuvula et al., 2007; Vogel et al., 2009). The evaluation of a synthetic SPL inhibitor, LX2931, is currently underway in phase II clinical trials in RA patients (Bagdanoff et al., 2010). 


\subsubsection{Depletion of extracellular S1P with S1P antibody}

Specific antibodies against S1P have been developed and are currently being tested in clinical studies for the treatment of cancer, fibrosis, inflammation, macular degeneration, diabetic retinopathy, glaucoma, and other diseases (Graler and Goetzl, 2004). The antibodies are thought to bind S1P and reduce the extracellular pool of bioactive S1P (Sabbadini, 2006). Indeed, a preclinical study using blocking S1P antibodies to prevent tumor progression was recently reported (Visentin et al., 2006). In this hallmark study, a specific monoclonal antibody recognizing S1P was administered to mice harboring human cancer xenografts. The intervention reduced, and in some cases completely eliminated, tumour formation and accompanying tumour angiogenesis. The results suggest that antibody-mediated inhibition of S1P signalling may be developed as a strategy for inhibiting pannus formation and angiogenesis in RA.

\subsection{Targeting S1P receptors}

S1P and its receptors are powerful regulators of various critical functions of RA synoviocytes. Targeting these cells with specific S1P receptor agonists or antagonists can modulate specific inflammatory responses locally and systemically by altering cell migration, cytokine and chemokine secretion, and proliferation/survival. RP-001, a potent S1P1 receptor agonist with properties similar to those reported for FTY-720 on S1P1 receptor degradation and lymphocyte sequestration from the blood, was recently characterized (Cahalan et al., 2011). The short half-life of RP-001 in vivo, however, disqualifies this compound for clinical use.

\subsubsection{S1P receptor agonists, FTY-720}

The immunosuppressant drug FTY-720 (fingolimod) was originally described as a sphingosine analogue that is phosphorylated in vivo by SphK2 to a S1P agonist for all S1P receptors except S1P2 (Brinkmann et al., 2002; Mandala et al., 2002). Further studies revealed that FTY-720-P not only activates S1P receptors, it also down-regulates them and consequently renders cells unresponsive to S1P (Matloubian et al., 2004). FTY-720 has been suggested to play a major role in autoimmune disease, such as multiple sclerosis [reviewed in (Nicholas et al., 2011)]. Multiple sclerosis is a common neurological disability, in which autoreactive T-cells migrate across the blood-brain barrier and attack myelin sheaths, leading to demyelination and axonal damage. FTY-720 deprives thymocytes and lymphocytes by downregulating S1P receptors and interfering with S1P signalling necessary for their egress from secondary lymphoid tissues (Cyster, 2005; Graler and Goetzl, 2004; Kappos et al., 2006; Matloubian et al., 2004). The results of a Phase II clinical trial evaluating the efficacy and safety of FTY-720 for treating relapsing multiple sclerosis showed that the annualized relapse rate of the FTY-720 group was significantly decreased (Kappos et al., 2006). FTY-720 was recently FDA-approved for the treatment of multiple sclerosis (Strader et al., 2011).

The effects of FTY-720 have been examined in several animal models of arthritis (Matsuura et al., 2000; Tsunemi et al., 2010; Wang et al., 2007). In the study of Wang et al. (2007), CIA rats were treated daily with FTY-720 for 28 days and the arthritis index was measured. Radiological analysis revealed that FTY-720-treated CIA rats had less joint damage in comparison to untreated CIA rats. Moreover, while histological assessment showed that CIA rats suffered from inflammatory cell infiltration and synovial hyperplasia in their joints, 
FTY-720 treatment clearly reduced these pathological parameters. Similarly, Matsuura et al. (2000) compared FTY-720 with two other anti-rheumatic compounds, mizoribine and prednisolone, in the rat models of CIA and AIA. FTY-720 completely suppressed the increase in hind paw volume and bone destruction to normal control levels by inhibiting leukocyte accumulation in the arthritic joint. Moreover, FTY-720 was shown to possess antiarthritic activity with a wider margin of safety in AIA and CIA models as compared to mizoribine and prednisolone. A recent study by Tsunmi et al. (2010) revealed that FTY-720 administration suppressed the progression of laminarin-induced arthritis in SKG mice. FTY720 treatment decreased IL- 6 and TNF- $\alpha$ expression by synovial fibroblasts, diminished the number of inflammatory cells migrating into the $\square$ joints, and suppressed bone destruction.

\subsubsection{S1P receptor antagonists}

Blocking S1P receptor activity by using S1P receptor antagonists may lead to promising therapeutic strategies for patients suffering from RA. Despite the lack of more comprehensive in vivo data, considerable progress has been made in the identification of the S1P receptors regulating synovial fibroblast migration, production of cytokines/chemokines and $\mathrm{PGE}_{2}$, proliferation, and survival. Several compounds targeting S1P receptors have also been used to decipher the biological roles of S1P receptors. The S1P1/3 antagonist VPC23019 and S1P3 antagonist CAY10444 blocked S1Pmediated synoviocyte migration and cytokine secretion, whereas the S1P2 antagonist JTE013 attenuated S1P-mediated cytokine secretion (Kitano et al., 2006; Zhao et al., 2008). The pharmacokinetics, bioavailability and metabolic characteristics of these S1P antagonists are essential to advance in vivo studies and for therapeutic intervention. Indeed, pharmacological approaches have been developed to block the action of S1P in the context of cancer progression (reviewed in Peyruchaud, 2009).

\section{Conclusion}

In summary, SphKs and S1P/S1PR signalling appear to play essential roles in modulating RA pathogenesis. The SphK1 pathway is activated and likely plays a pro-inflammatory role in mouse models of inflammatory arthritis. It is fascinating that the blockade of SphK1 activity results in the simultaneous reduction of several inflammatory responses such as pro-inflammatory cytokines and inflammatory cell infiltration into the synovium. Excessive S1P and enhanced S1P receptor expression are detected in the synovium of RA patients. S1P and signalling through S1P receptors induce expression of inflammatory cytokines and suppress apoptosis of B lymphoblastoid cells and fibroblast-like synoviocytes. Although the possible mechanism by which S1P exerts its activity in RA remains to be fully characterized, further understanding of S1P metabolism and S1P receptor expression by synovial tissues represents an exploitable objective for the development of novel chemotherapeutic agents in the treatment of RA.

\section{Acknowledgment}

The authors would like to thank Ms. Lynn Davis for her editorial assistance. The works cited in the review from the authors' laboratory were supported by research grants from the Canadian Arthritis Network and the Arthritis Society of Canada. 


\section{References}

Alvarez, S. E., et al., 2007. Autocrine and paracrine roles of sphingosine-1-phosphate. Trends Endocrinol Metab. 18, 300-7.

An, S., et al., 1997. Identification of cDNAs encoding two G protein-coupled receptors for lysosphingolipids. FEBS Lett. 417, 279-82.

Ancellin, N., Hla, T., 1999. Differential pharmacological properties and signal transduction of the sphingosine 1-phosphate receptors EDG-1, EDG-3, and EDG-5. J Biol Chem. 274, 18997-9002.

Bagdanoff, J. T., et al., 2010. Inhibition of sphingosine 1-phosphate lyase for the treatment of rheumatoid arthritis: discovery of (E)-1-(4-((1R,2S,3R)-1,2,3,4-tetrahydroxybutyl)1H-imidazol-2-yl)ethanone oxime (LX2931) and (1R,2S,3R)-1-(2-(isoxazol-3-yl)-1Himidazol-4-yl)butane-1,2,3,4-tetraol (LX2932). J Med Chem. 53, 8650-62.

Bagdanoff, J. T., et al., 2009. Inhibition of sphingosine-1-phosphate lyase for the treatment of autoimmune disorders. J Med Chem. 52, 3941-53.

Baker, D. A., et al., 2010. Genetic sphingosine kinase 1 deficiency significantly decreases synovial inflammation and joint erosions in murine TNF-alpha-induced arthritis. J Immunol. 185, 2570-9.

Bandhuvula, P., et al., 2007. A rapid fluorescence assay for sphingosine-1-phosphate lyase enzyme activity. J Lipid Res. 48, 2769-78.

Ben-Av, P., et al., 1995. Induction of vascular endothelial growth factor expression in synovial fibroblasts by prostaglandin E and interleukin-1: a potential mechanism for inflammatory angiogenesis. FEBS Lett. 372, 83-7.

Billich, A., et al., 2005. Basal and induced sphingosine kinase 1 activity in A549 carcinoma cells: function in cell survival and IL-1beta and TNF-alpha induced production of inflammatory mediators. Cell Signal. 17, 1203-17.

Blom, T., et al., 2005. Enhancement of intracellular sphingosine-1-phosphate production by inositol 1,4,5-trisphosphate-evoked calcium mobilisation in HEK-293 cells: endogenous sphingosine-1-phosphate as a modulator of the calcium response. Cell Signal. 17, 827-36.

Bonhoure, E., et al., 2006. Overcoming MDR-associated chemoresistance in HL-60 acute myeloid leukemia cells by targeting sphingosine kinase-1. Leukemia. 20, 95-102.

Brinkmann, V., et al., 2002. The immune modulator FTY720 targets sphingosine 1-phosphate receptors. J Biol Chem. 277, 21453-7.

Cahalan, S. M., et al., 2011. Actions of a picomolar short-acting S1P agonist in S1P-eGFP knock-in mice. Nat Chem Biol. 7, 254-6.

Choi, J. W., et al., 2008. Biological roles of lysophospholipid receptors revealed by genetic null mice: an update. Biochim Biophys Acta. 1781, 531-9.

Chun, J., et al., 2002. International Union of Pharmacology. XXXIV. Lysophospholipid receptor nomenclature. Pharmacol Rev. 54, 265-9.

Coursol, S., et al., 2005. Arabidopsis sphingosine kinase and the effects of phytosphingosine1-phosphate on stomatal aperture. Plant Physiol. 137, 724-37.

Cyster, J. G., 2005. Chemokines, sphingosine-1-phosphate, and cell migration in secondary lymphoid organs. Annu Rev Immunol. 23, 127-59.

Delon, C., et al., 2004. Sphingosine kinase 1 is an intracellular effector of phosphatidic acid. J Biol Chem. 279, 44763-74.

Feldmann, M., et al., 1996a. Rheumatoid arthritis. Cell. 85, 307-10. 
Feldmann, M., et al., 1996b. Role of cytokines in rheumatoid arthritis. Annu Rev Immunol. 14, 397-440.

Fitzpatrick, L. R., et al., 2011. Attenuation of arthritis in rodents by a novel orally-available inhibitor of sphingosine kinase. Inflammopharmacology. 19, 75-87.

French, K. J., et al., 2006. Antitumor activity of sphingosine kinase inhibitors. J Pharmacol Exp Ther. 318, 596-603.

Gamble, J. R., et al., 2006. Phenoxodiol, an experimental anticancer drug, shows potent antiangiogenic properties in addition to its antitumour effects. Int J Cancer. 118, 2412-20.

Garcia, J. G., et al., 2001. Sphingosine 1-phosphate promotes endothelial cell barrier integrity by Edg-dependent cytoskeletal rearrangement. J Clin Invest. 108, 689-701.

Gardell, S. E., et al., 2006. Emerging medicinal roles for lysophospholipid signaling. Trends Mol Med. 12, 65-75.

Ghosh, M., et al., 2004. Role of cytosolic phospholipase $\mathrm{A}(2)$ in prostaglandin $\mathrm{E}(2)$ production by lung fibroblasts. Am J Respir Cell Mol Biol. 30, 91-100.

Graler, M. H., Goetzl, E. J., 2004. The immunosuppressant FTY720 down-regulates sphingosine 1-phosphate G-protein-coupled receptors. FASEB J. 18, 551-3.

Hait, N. C., et al., 2009. Regulation of histone acetylation in the nucleus by sphingosine-1phosphate. Science. 325, 1254-7.

Hidaka, T., et al., 2001. Dynamic changes in cytokine levels in serum and synovial fluid following filtration leukocytapheresis therapy in patients with rheumatoid arthritis. J Clin Apher. 16, 74-81.

Hla, T., 2004. Physiological and pathological actions of sphingosine 1-phosphate. Semin Cell Dev Biol. 15, 513-20.

Huwiler, A., Pfeilschifter, J., 2008. New players on the center stage: sphingosine 1-phosphate and its receptors as drug targets. Biochem Pharmacol. 75, 1893-900.

Im, D. S., et al., 2000. Characterization of a novel sphingosine 1-phosphate receptor, Edg-8. J Biol Chem. 275, 14281-6.

Ishii, I., et al., 2004. Lysophospholipid receptors: signaling and biology. Annu Rev Biochem. $73,321-54$.

Ishii, M., et al., 2009. Sphingosine-1-phosphate mobilizes osteoclast precursors and regulates bone homeostasis. Nature. 458, 524-8.

Jenkins, G. M., Hannun, Y. A., 2001. Role for de novo sphingoid base biosynthesis in the heat-induced transient cell cycle arrest of Saccharomyces cerevisiae. J Biol Chem. $276,8574-81$.

Johnson, K. R., et al., 2002. PKC-dependent activation of sphingosine kinase 1 and translocation to the plasma membrane. Extracellular release of sphingosine-1phosphate induced by phorbol 12-myristate 13-acetate (PMA). J Biol Chem. 277, 35257-62.

Kamada, K., et al., 2009. Expression of sphingosine kinase 2 in synovial fibroblasts of rheumatoid arthritis contributing to apoptosis by a sphingosine analogue, FTY720. Pathol Int. 59, 382-9.

Kappos, L., et al., 2006. Oral fingolimod (FTY720) for relapsing multiple sclerosis. N Engl J Med. 355, 1124-40.

Kim, M. K., et al., 2006. Sphingosine-1-phosphate stimulates rat primary chondrocyte proliferation. Biochem Biophys Res Commun. 345, 67-73. 
Kitano, M., et al., 2006. Sphingosine 1-phosphate/sphingosine 1-phosphate receptor 1 signaling in rheumatoid synovium: regulation of synovial proliferation and inflammatory gene expression. Arthritis Rheum. 54, 742-53.

Kluk, M. J., Hla, T., 2002. Signaling of sphingosine-1-phosphate via the S1P/EDG-family of G-protein-coupled receptors. Biochim Biophys Acta. 1582, 72-80.

Knedla, A., et al., 2007. Developments in the synovial biology field 2006. Arthritis Res Ther. 9, 209.

Kobayashi, N., et al., 2006. Sphingosine 1-phosphate is released from the cytosol of rat platelets in a carrier-mediated manner. J Lipid Res. 47, 614-21.

Koch, A. E., 2005. Chemokines and their receptors in rheumatoid arthritis: future targets? Arthritis Rheum. 52, 710-21.

Kohama, T., et al., 1998. Molecular cloning and functional characterization of murine sphingosine kinase. J Biol Chem. 273, 23722-8.

Lai, W. Q., et al., 2008. Anti-inflammatory effects of sphingosine kinase modulation in inflammatory arthritis. J Immunol. 181, 8010-7.

Lai, W. Q., et al., 2009. Distinct roles of sphingosine kinase 1 and 2 in murine collageninduced arthritis. J Immunol. 183, 2097-103.

Lee, M. J., et al., 1999. Vascular endothelial cell adherens junction assembly and morphogenesis induced by sphingosine-1-phosphate. Cell. 99, 301-12.

Lee, M. J., et al., 2001. Akt-mediated phosphorylation of the G protein-coupled receptor EDG-1 is required for endothelial cell chemotaxis. Mol Cell. 8, 693-704.

Lee, M. J., et al., 1998. Sphingosine-1-phosphate as a ligand for the G protein-coupled receptor EDG-1. Science. 279, 1552-5.

Leroux, M. E., et al., 2007. Sphingolipids and the sphingosine kinase inhibitor, SKI II, induce BCL-2-independent apoptosis in human prostatic adenocarcinoma cells. Prostate. 67, 1699-717.

Lettesjo, H., et al., 1998. Synovial fluid cytokines in patients with rheumatoid arthritis or other arthritic lesions. Scand J Immunol. 48, 286-92.

Limaye, V., et al., 2009. Chronic increases in sphingosine kinase-1 activity induce a proinflammatory, pro-angiogenic phenotype in endothelial cells. Cell Mol Biol Lett. 14, 424-41.

Lo, C. G., et al., 2005. Cyclical modulation of sphingosine-1-phosphate receptor 1 surface expression during lymphocyte recirculation and relationship to lymphoid organ transit. J Exp Med. 201, 291-301.

Maceyka, M., et al., 2005. SphK1 and SphK2, sphingosine kinase isoenzymes with opposing functions in sphingolipid metabolism. J Biol Chem. 280, 37118-29.

Mandala, S., et al., 2002. Alteration of lymphocyte trafficking by sphingosine-1-phosphate receptor agonists. Science. 296, 346-9.

Martel-Pelletier, J., et al., 2003. Cyclooxygenase-2 and prostaglandins in articular tissues. Semin Arthritis Rheum. 33, 155-67.

Masuko, K., et al., 2007. Sphingosine-1-phosphate attenuates proteoglycan aggrecan expression via production of prostaglandin E2 from human articular chondrocytes. BMC Musculoskelet Disord. 8, 29.

Matloubian, M., et al., 2004. Lymphocyte egress from thymus and peripheral lymphoid organs is dependent on S1P receptor 1. Nature. 427, 355-60. 
Matsuura, M., et al., 2000. Effect of FTY720, a novel immunosuppressant, on adjuvant- and collagen-induced arthritis in rats. Int J Immunopharmacol. 22, 323-31.

McInnes, I. B., Schett, G., 2007. Cytokines in the pathogenesis of rheumatoid arthritis. Nat Rev Immunol. 7, 429-42.

Mechtcheriakova, D., et al., 2007. Sphingosine 1-phosphate phosphatase 2 is induced during inflammatory responses. Cell Signal. 19, 748-60.

Merrill, A. H., Jr., et al., 1997. Sphingolipids--the enigmatic lipid class: biochemistry, physiology, and pathophysiology. Toxicol Appl Pharmacol. 142, 208-25.

Mino, T., et al., 1998. Interleukin-1alpha and tumor necrosis factor alpha synergistically stimulate prostaglandin E2-dependent production of interleukin-11 in rheumatoid synovial fibroblasts. Arthritis Rheum. 41, 2004-13.

Mitra, P., et al., 2006. Role of ABCC1 in export of sphingosine-1-phosphate from mast cells. Proc Natl Acad Sci U S A. 103, 16394-9.

Muller-Ladner, U., et al., 1996. Synovial fibroblasts of patients with rheumatoid arthritis attach to and invade normal human cartilage when engrafted into SCID mice. Am J Pathol. 149, 1607-15.

Nicholas, R., et al., 2011. Development of oral immunomodulatory agents in the management of multiple sclerosis. Drug Des Devel Ther. 5, 255-74.

Nochi, H., et al., 2008. Stimulatory role of lysophosphatidic acid in cyclooxygenase-2 induction by synovial fluid of patients with rheumatoid arthritis in fibroblast-like synovial cells. J Immunol. 181, 5111-9.

Olsen, N. J., Stein, C. M., 2004. New drugs for rheumatoid arthritis. N Engl J Med. 350, 216779.

Ospelt, C., et al., 2004. Synovial activation in rheumatoid arthritis. Front Biosci. 9, 2323-34.

Paugh, S. W., et al., 2008. A selective sphingosine kinase 1 inhibitor integrates multiple molecular therapeutic targets in human leukemia. Blood. 112, 1382-91.

Peyruchaud, O., 2009. Novel implications for lysophospholipids, lysophosphatidic acid and sphingosine 1-phosphate, as drug targets in cancer. Anticancer Agents Med Chem. 9, 381-91.

Pi, X., et al., 2006. Sphingosine kinase 1-mediated inhibition of Fas death signaling in rheumatoid arthritis B lymphoblastoid cells. Arthritis Rheum. 54, 754-64.

Pitson, S. M., et al., 2005. Phosphorylation-dependent translocation of sphingosine kinase to the plasma membrane drives its oncogenic signalling. J Exp Med. 201, 49-54.

Pyne, S., Pyne, N. J., 2000. Sphingosine 1-phosphate signalling in mammalian cells. Biochem J. 349, 385-402.

Rosen, H., et al., 2003. Rapid induction of medullary thymocyte phenotypic maturation and egress inhibition by nanomolar sphingosine 1-phosphate receptor agonist. Proc Natl Acad Sci U S A. 100, 10907-12.

Rosen, H., et al., 2009. Sphingosine 1-Phosphate Receptor Signaling. Annu Rev Biochem.

Rosenfeldt, H. M., et al., 2001. EDG-1 links the PDGF receptor to Src and focal adhesion kinase activation leading to lamellipodia formation and cell migration. FASEB J. 15, 2649-59.

Saba, J. D., Hla, T., 2004. Point-counterpoint of sphingosine 1-phosphate metabolism. Circ Res. 94, 724-34.

Sabbadini, R. A., 2006. Targeting sphingosine-1-phosphate for cancer therapy. Br J Cancer. 95, 1131-5. 
Sanchez, T., Hla, T., 2004. Structural and functional characteristics of S1P receptors. J Cell Biochem. 92, 913-22.

Sato, K., et al., 2007. Critical role of ABCA1 transporter in sphingosine 1-phosphate release from astrocytes. J Neurochem. 103, 2610-9.

Schwab, S. R., et al., 2005. Lymphocyte sequestration through S1P lyase inhibition and disruption of S1P gradients. Science. 309, 1735-9.

Shiozawa, S., et al., 1983. Morphologic observations in the early phase of the cartilagepannus junction. Light and electron microscopic studies of active cellular pannus. Arthritis Rheum. 26, 472-8.

Spiegel, S., Milstien, S., 2003. Sphingosine-1-phosphate: an enigmatic signalling lipid. Nat Rev Mol Cell Biol. 4, 397-407.

Strader, C. R., et al., 2011. Fingolimod (FTY720): A Recently Approved Multiple Sclerosis Drug Based on a Fungal Secondary Metabolite. J Nat Prod. 74, 900-7.

Sugimoto, N., et al., 2003. Inhibitory and stimulatory regulation of Rac and cell motility by the G12/13-Rho and Gi pathways integrated downstream of a single G proteincoupled sphingosine-1-phosphate receptor isoform. Mol Cell Biol. 23, 1534-45.

Taha, T. A., et al., 2004. Sphingosine-1-phosphate receptors: receptor specificity versus functional redundancy. Biochim Biophys Acta. 1682, 48-55.

Taha, T. A., et al., 2006. Sphingosine kinase: biochemical and cellular regulation and role in disease. J Biochem Mol Biol. 39, 113-31.

Takabe, K., et al., 2010. Estradiol induces export of sphingosine 1-phosphate from breast cancer cells via ABCC1 and ABCG2. J Biol Chem. 285, 10477-86.

Takabe, K., et al., 2008. "Inside-out" signaling of sphingosine-1-phosphate: therapeutic targets. Pharmacol Rev. 60, 181-95.

Tsunemi, S., et al., 2010. Effects of the novel immunosuppressant FTY720 in a murine rheumatoid arthritis model. Clin Immunol. 136, 197-204.

Van Brocklyn, J. R., et al., 2000. Sphingosine-1-phosphate is a ligand for the G proteincoupled receptor EDG-6. Blood. 95, 2624-9.

Van Brocklyn, J. R., et al., 1998. Dual actions of sphingosine-1-phosphate: extracellular through the Gi-coupled receptor Edg-1 and intracellular to regulate proliferation and survival. J Cell Biol. 142, 229-40.

Visentin, B., et al., 2006. Validation of an anti-sphingosine-1-phosphate antibody as a potential therapeutic in reducing growth, invasion, and angiogenesis in multiple tumor lineages. Cancer Cell. 9, 225-38.

Vogel, P., et al., 2009. Incomplete inhibition of sphingosine 1-phosphate lyase modulates immune system function yet prevents early lethality and non-lymphoid lesions. PLoS One. 4, e4112.

Wang, F., et al., 2007. Reduction of CD4 positive T cells and improvement of pathological changes of collagen-induced arthritis by FTY720. Eur J Pharmacol. 573, 230-40.

Xia, P., et al., 2000. An oncogenic role of sphingosine kinase. Curr Biol. 10, 1527-30.

Yamazaki, Y., et al., 2000. Edg-6 as a putative sphingosine 1-phosphate receptor coupling to $\mathrm{Ca}(2+)$ signaling pathway. Biochem Biophys Res Commun. 268, 583-9.

Zhang, Z., Schluesener, H. J., 2007. FTY720: a most promising immunosuppressant modulating immune cell functions. Mini Rev Med Chem. 7, 845-50.

Zhao, C., et al., 2008. Specific and overlapping sphingosine-1-phosphate receptor functions in human synoviocytes: impact of TNF-alpha. J Lipid Res. 49, 2323-37. 


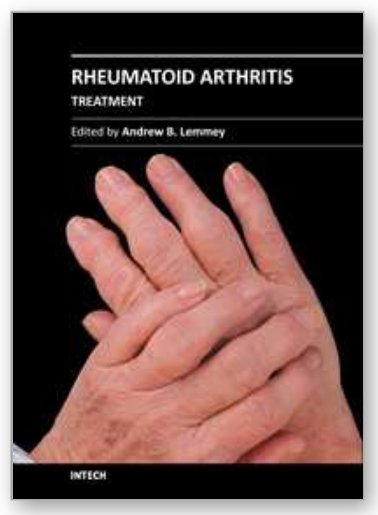

\author{
Rheumatoid Arthritis - Treatment \\ Edited by Dr. Andrew Lemmey
}

ISBN 978-953-307-850-2

Hard cover, 366 pages

Publisher InTech

Published online 18, January, 2012

Published in print edition January, 2012

The purpose of this book is to provide up-to-date, interesting, and thought-provoking perspectives on various aspects of research into current and potential treatments for rheumatoid arthritis (RA). This book features 17 chapters, with contributions from numerous countries (e.g. UK, USA, Canada, Japan, Sweden, Turkey, Bosnia and Herzegovina, Slovakia), including chapters from internationally recognized leaders in rheumatology research. It is anticipated that Rheumatoid Arthritis - Treatment will provide both a useful reference and source of potential areas of investigation for research scientists working in the field of RA and other inflammatory arthropathies.

\title{
How to reference
}

In order to correctly reference this scholarly work, feel free to copy and paste the following:

Sylvain G. Bourgoin, Chenqi Zhao and Maria J. Fernandes (2012). Targeting the Metabolism and Receptors of Sphingosine-1- Phosphate for the Treatment of Rheumatoid Arthritis, Rheumatoid Arthritis - Treatment, Dr. Andrew Lemmey (Ed.), ISBN: 978-953-307-850-2, InTech, Available from:

http://www.intechopen.com/books/rheumatoid-arthritis-treatment/targeting-the-metabolism-and-receptors-ofsphingosine-1-phosphate-for-the-treatment-of-rheumatoid-ar

\section{INTECH}

open science | open minds

\section{InTech Europe}

University Campus STeP Ri

Slavka Krautzeka 83/A

51000 Rijeka, Croatia

Phone: +385 (51) 770447

Fax: +385 (51) 686166

www.intechopen.com

\section{InTech China}

Unit 405, Office Block, Hotel Equatorial Shanghai

No.65, Yan An Road (West), Shanghai, 200040, China

中国上海市延安西路65号上海国际贵都大饭店办公楼405单元

Phone: +86-21-62489820

Fax: $+86-21-62489821$ 
(C) 2012 The Author(s). Licensee IntechOpen. This is an open access article distributed under the terms of the Creative Commons Attribution 3.0 License, which permits unrestricted use, distribution, and reproduction in any medium, provided the original work is properly cited. 